\title{
Bone Marrow Involvement in Non Hematological Disorders - An Institutional Experience
}

\author{
Dr. R Panigrahi, Dr. P K Behera, Dr. K P Tripathy. \\ ${ }^{1}$ Associate Professor, Department of Pathology, Kalinga Institute of Medical Sciences, \\ Bhubaneswar, 751024 \\ ${ }^{2}$ Associate Professor, Department of Medicine, Kalinga Institute of Medical Sciences, \\ Bhubaneswar, 751024 \\ ${ }^{3}$ Professor, Department of Medicine, Kalinga Institute of Medical Sciences, Bhubaneswar.
}

\begin{abstract}
Objective: To find out the frequency of involvement of bone marrow by non - haematological disorders in our institution and to evaluate the role of bone marrow aspiration and biopsy in diagnosing these disorders. Materials \& Methods: It was a retrospective study that extended from January 2013 to December 2016. A total of 339 bone marrow aspirates and trephine biopsies were performed during this period. Only the non haematological disorders were studied and their clinical and laboratory parameters were recorded. Peripheral blood picture was studied. Bone marrow aspiration smears \& biopsies were evaluated.

Results: During the study period, 29 patients were diagnosed to have non-haematological disorders which constituted $8.5 \%$ of the total patients who had undergone bone marrow examination. The age of patients at time of procedure ranged from 3 years to 84years, with male to female ratio of $2.2: 1$. Anemia was the most frequent clinical finding followed by fever and weight loss. In adults, metastatic tumors were the commonest non hematological condition diagnosed on bone marrow examination. In children also, metastatic tumors were the commonest non-hematological disease.

Conclusion: Metastatic solid tumors were the frequent non-hematologic disorders involving bone marrow in both adults and pediatric patients . Hence, bone marrow examination can be a safe, easy and useful laboratory tool in the diagnosis of various metastatic malignancies and other disorders like granulomatous lesions.
\end{abstract}

Keywords: Bone marrow examination, non hematological disorders , metastatic tumors.

\section{Introduction}

Although bone marrow examination is a common procedure in the evaluation of patients with cancer and other non-haematologic disorders, its role and contribution have been questioned in recent years.Metastatic involvement with tumors ${ }^{[4]}$,granulomatous diseases ${ }^{[3]}$, storage disorders ${ }^{[10],}$ haemophagocytic Syndrome ${ }^{[6,11]}$, histiocytoses ${ }^{[7]}$ and Leishmaniasis ${ }^{[8,9]}$ can be diagnosed through bone marrow examination. In addition to hematological malignancies; bone marrow examination has been increasingly useful in documenting metastatic involvement of tumors.${ }^{[4]}$ The present study was carried out to find out the frequency of non hematological conditions which were diagnosed by bone marrow aspiration and biopsy in our institution so that the results will help the clinicians in selecting cases for bonemarrow examination for proper evaluation of their patients.

\section{Aim \& Objective}

To determine the frequency of involvement of bone marrow by non hematological disorders.

\section{Materials \& Methods}

The study was a retrospective study conducted over a period of 4 years from January 2013 to December 2016, in the hematology section of the Department of Pathology. A total of 339 bone marrow aspirations and trephines were performed during this period according to the standard techniques described in text books. Of these, 29 patients were diagnosed to have non hematological disorders from bonemarrow examination and constituted our study group. We analyzed and recorded the brief clinical history, clinical presentation, complete blood count of these patients. Peripheral blood picture, bone marrow aspiration smears and trephine biopsied were reviewed . The patients included both adults \& children of both sexes. The smears prepared from concentrated marrow cells were routinely stained with Leishman stain while biopsy specimens were decalcified and paraffin embedded blocks were prepared and sections stained with the usual haematoxylin and eosin (H\&E) stain. Reticulin, Ziehl-Neelsen and appropriate marrow histochemical stains were used where considered necessary.

\section{Results}


The present study was a retrospective study done over a period of 4 years and was conducted in the hematology section of the Department of Pathology in our institution. A total number of 339 bone marrow aspirations \& trephine biopsies were performed for various clinical indications during the study period. Of these cases , 29 ( $8.5 \%$ ) patients were diagnosed to have non- hematolological disorders on bone marrow examination and were included in our study.

The study group included 20 males and 9 females with a M:F ratio of 2.2:1. Both children and adults were included in the study group. The age of the patients ranged from 3 years to 84 years with a median age of 55 years at presentation. Maximum number of $11(37.9 \%)$ patients belonged to the age group of 51 to60 years.

Table 1 - Age \& sex distribution of the patients $(\mathrm{n}=29)$

\begin{tabular}{|l|l|l|l|l|}
\hline Age group (years) & No .of cases & Percentage (\%) & Males & Females \\
\hline $0-10$ & 4 & 13.8 & 3 & 1 \\
\hline $11-20$ & 1 & 3.5 & 1 & - \\
\hline $21-30$ & 3 & 10.3 & 3 & - \\
\hline $31-40$ & 1 & 3.5 & 1 & - \\
\hline $41-50$ & 1 & 3.5 & - & 1 \\
\hline $51-60$ & 11 & 37.9 & 7 & 4 \\
\hline $61-70$ & 2 & 6.8 & 1 & 1 \\
\hline $71-80$ & 5 & 17.2 & 4 & 1 \\
\hline $81-90$ & 1 & 3.5 & - & 1 \\
\hline Total & 29 & 100 & 20 & 9 \\
\hline
\end{tabular}

Of the total 29 cases, 25 patients were adults and only 4 patients belonged to the pediatric age group. Metastatic tumors to the marrow was the most common non-hematological disease entity diagnosed in 11 cases, which constituted about $37.9 \%$ of patients .

Table 2 - Frequency of bone marrow involvement in non hematological diseases in children and adults $(\mathrm{n}=29)$

\begin{tabular}{|l|l|l|l|l|}
\hline Disease & No. of cases & Percentage (\%) & Age > 15 years & Age < 15 years \\
\hline Metastatic tumors & 11 & 37.9 & 9 & 2 \\
\hline Granulomatous lesion & 3 & & & \\
& & 10.3 & 3 & - \\
\hline Myeloid hyperplasia & 8 & 27.6 & 8 & - \\
\hline Hemophagocytosis & 3 & 10.3 & 2 & 1 \\
\hline Miscelleneous & 3 & 10.3 & 2 & 1 \\
\hline Parvo virus infection & 1 & 3.5 & 1 & - \\
\hline Total & 29 & 100 & 25 & 4 \\
\hline
\end{tabular}

\section{Discussion}

Although bone marrow biopsy is an uncomfortable procedure for the patient and should be performed only when there is a clear clinical indication ${ }^{[1]}$ in most cases it can be done as an easy an cheap invasive procedure in the diagnosis of many hematological \& non hematological disorders. Both marrow aspiration and trephine biopsy can be performed with little patient discomfort, provided adequate local anesthesia has been used and the patient is co-operative.

The present study was done find out the frequency of various non hematological diseases which were diagnosed on bone marrow examination in our institution during a period of 4 years . Comparable data regarding similar studies in literature is scarce. Few studies do support do support the role of bone marrow examination in the diagnosis of different non-hematological diseases. ${ }^{[5,14]}$ Various studies have been conducted on the involvement of marrow by metastatic solid tumors. ${ }^{[4]}$

The study group constituted 29 patients $(8.5 \%)$ of the total patients who had undergone bonemarrow examination for various indications. In a similar study by Syed et al , 63 patients (1.4\%) were diagnosed to have non-haematological diseases detected primarily through bone marrow examination. The mean age of patients at time of procedure was 33years (range 6 months to 89 years), with male to female ratio of $3.2: 1^{[5]}$.

In our study, anemia was the most frequent clinical presentation. Various studies reveal an incidence of anemia in neoplastic diseases from $55-90 \%$ of cases ${ }^{[12,13]}$ but the same cannot be compared for other diseases because of the non availability of equivalent data. The other clinical findings were weight loss, fever and backache.Bone marrow aspirate helped us to detect only few of all the metastatic tumours that were diagnosed . Most of the cases showed a dry tap on aspiration or yielded only very scanty material for opinion. In these cases bone marrow trephine biopsy imprint smears and the biopsy proved more useful in reaching at a diagnosis Thus, the demonstration of tumour metastases by bone marrow biopsy was superior to the bone marrow aspirate in our study. Similar observations were made by others as well. ${ }^{[12,14]}$ Nevertheless, trephine biopsy and 
bone marrow aspirate should be regarded as complementary investigation because either might show tumour cells when other procedure had failed to do so. ${ }^{[15]}$

The bone marrow is commonly involved by metastatic tumors, this is because it has a good blood supply with lots of nutrients and growth factors, making it a fertile place for tumor cells to grow. ${ }^{[1,2]}$ In addition to hematological malignancies; bone marrow examination has been increasingly useful in documenting metastatic involvement of tumors ${ }^{[4]}$ The incidence of metastatic disease to bone marrow varies among different authors. In the present study metastatic adenocarcinoma was present in 5 cases. The primary site were searched for and was found to be breast ( 2 cases) and GIT ( 1 case). Rest of the cases were lost to follow up, so the primary site could not be ascertained and however, it was out of the scope of our study. Metastatic tumors were the most common non hematological disorder diagnosed in adults ( $31 \%$ ) which was comparable to the study by Syed et al ${ }^{[5]}$.

In our study there were only 4 patients in the pediatric age group. 2 cases $(6.9 \%)$ of metastatic neuroblastoma and one case of hemophagocytosis was diagnosed. In children, in their study by Syed et al ${ }^{[5]}$, storage disorders were more prevalent followed by haemophagocytosis as the second commonest nonhaaematological disease. Granulomatous reactions can be seen in tuberculosis, sarcoidosis, fungal infection and Hodgkin's lymphoma. In our study there were 3 cases (10.3\%) which showed granulomas on trephine biopsy. In our country as systemic tuberculosis is more prevalent, Ziehl Neelsen staining was done in all cases to exclude tuberculosis. 2 out of 3 such cases were positive for acid fast bacilli. In the study by Syed et al ${ }^{[5]}$ ,granulomatous lesions were the second most common disease entity diagnosed in adults.

\section{Conclusion}

In our study, we have concluded that bone marrow examination can be useful in diagnosing non hematological conditions like metastatic tumors, granulomatous diseases, and many other miscellaneous diseases . In many cases trephine biopsy proves to be more helpful and at times complimentary to aspiration as a diagnostic method in these conditions. Bone marrow examination can be used as a diagnostic tool in the diagnosis of various non hematological diseases in addition to hematological disorders and malignancies.

\section{References}

[1]. Bain BJ. Bone marrow trephine biopsy. J Clin Pathol 2001; 54: 737-42.

[2]. John R. Krause. Metastatic tumours in Bone marrow biopsy. 1981. p 188-95.

[3]. Kumar PV, Monabati A, Kadivar R, Solemanfour H. Peripheral blood and marrow findings in disseminated bacilli calmette-Guerin infection. J Pediatr Hematol Oncol 2005; 27: 97-9.

[4]. Mohanty SK, Dash S. Bone marrow metastasis in solid tumors. Indian J Pathol Microbiol 2003 Oct;46(4): 613-16.

[5]. Syed NN, Moiz BB, Adil SN, Khurshid M. Diagnostic importance of bone marrow examination in non-hematological disorders. J Pak Med Assoc 2007;57:123-25.

[6]. Tsuda H. Haemophagocytic syndrome (HPS) in children and adults. Int J Hematol 1997; 65:215-26.

[7]. Stephan JL. Histiocytoses. Eur J Pediatr. 1995; 154:600-9.Pathol. 2003; 20:226-36.

[8]. Sipahi T, Tavil B, Oksal A. Visceral leishmaniasis and pseudomonas septicemia associated with haemophagocytic syndrome and myelodysplasia in a Turkish child. Turk J Pediatr 2005; 47: 191-4.

[9]. Magill AJ, Grogl M, Gasser RA Jr, Sun W, Orter LN. Visceral Infection Caused by Leishmania tropica in Veterans of Operation Desert Storm. New Engl J Med 1993; 328: 1383-7.

[10]. Chang KL, Gaal KK, Huang Q, Weiss LM. Histiocytic lesions involving the bone marrow. Semin Diagn Pathol. 2003; 20:226-36.

[11]. Stéphan JL, Koné-Paut I, Galambrun C, Mouy R, Bader-Meunier B, Preur AM. Reactive haemophagocytic syndrome in children with inflammatory disorders. A retrospective study of 24 patients. Rheumatology 2001; 40: 1285-92.

[12]. Contreras E, Ellis LD, Lee RE. Value of the bone marrow biopsy in the diagnosis of metastatic carcinoma. Cancer 1972; 29: 77883.

[13]. Hyman GA, Harvey JE. The pathogenesis of anemia in patients with carcinoma. Am J Med 1955; 19: 350-56.

[14]. Moid F, DePalma L. Comparison of relative value of bone marrow aspirates and bone marrow trephine biopsies in the diagnosis of solid tumor metastasis and hodgkin's lymphoma: institutional experience and literature review. Arch Pathol Lab Med 2005; 129: 497-501.

[15]. Anner RM, Drewinko B. Frequency and significance of bone marrow involvement by metastatic solid tumors. Cancer 1977; 39: 1337 